\title{
Field-Induced Quantum Criticality of Systems with Ferromagnetically Coupled Structural Spin Units
}

\author{
I. Rabuffo ${ }^{a, *}$, M. T. Mercaldo ${ }^{a}$, L. De $^{*}$ Cesare $^{a}$, \\ A. Caramico D'Auria ${ }^{\text {, }}$ \\ a Dipartimento di Fisica "E. R. Caianiello", Università di Salerno and CNISM, \\ Unitá di Salerno, I-84081 Baronissi (Salerno), Italy \\ b Dipartimento di Scienze Fisiche, Università di Napoli Federico II and \\ "Coherentia" CNR-INFM, I-80125 Napoli, Italy
}

\begin{abstract}
The field-induced quantum criticality of compounds with ferromagnetically coupled structural spin units (as dimers and ladders) is explored by applying Wilson's renormalization group framework to an appropriate effective action. We determine the low-temperature phase boundary and the behavior of relevant quantities decreasing the temperature with the applied magnetic field fixed at its quantum critical point value. In this context, a plausible interpretation of some recent experimental results is also suggested.
\end{abstract}

Key words: Anisotropic Ferromagnetic Heisenberg model, Quantum Phase Transitions, Dimers, Ladders.

PACS: 05.70.Fh; 64.60.Fr; 05.50.+q 
The study of quantum phase transitions (QPT's) and low-temperature properties close to a quantum critical point (QCP) of a wide variety of materials has recently attracted considerable attention and constitutes today a topical subject in condensed matter physics [1].

The main methods of tuning a system toward a QCP are essentially based on manipulation of doping, pressure and magnetic field. A lot of experiments on spin compounds has shown that the magnetic field is the most convenient non-thermal parameter to control the distance from a QCP.

Emerging magnetic field-induced QPT's have been observed in quantum antiferromagnetic (AFM) compounds as $\mathrm{KCuCl}_{3}$ [2], $\mathrm{TlCuCl}_{3}$ [2,3], $\mathrm{BaCuSi}_{2} \mathrm{O}_{6}$ [4], and $\mathrm{Cu}_{2}\left(\mathrm{C}_{5} \mathrm{H}_{12} \mathrm{~N}_{2}\right)_{2} \mathrm{Cl}_{4}$ [5] which consist of weakly coupled low-dimensional structural spin units such as dimers $[2,3,4]$ or ladders $[5,6]$. In absence of a magnetic field, these systems exhibit a gap between a singlet ground state and the lowest triplet excitation. When the field is switched on and increased, the gap decreases by Zeeman effect and a field-induced QPT occurs at a critical field which measures the amplitude of the original gap.

Recently, also the case of weakly ferromagnetically coupled spin units, although less explored then the AFM one, has attracted a lot of interest $[7,8,9,10,11,12]$ motivated by the possible existence of dimer and ladder materials with effective ferromagnetic (FM) interactions between the basic spin units $[7,9,12,13,14]$. Inelastic neutron scattering (INS) investigations on the spin dimer compounds $\mathrm{Cs}_{3} \mathrm{Cr}_{2} \mathrm{Br}_{9}$ [7], on the layered cuprate $\mathrm{Sr}_{14} \mathrm{Cu}_{24} \mathrm{O}_{41}$ [12] and on $\mathrm{CaV}_{2} \mathrm{O}_{5}$ [11], which contains layers of coupled two-leg ladders, appear particularly meaning-

\footnotetext{
* Corresponding author: Tel.: +39 089965 392; Fax: +39 089965 275;

E-mail address: rabuffo@sa.infn.it
} 
ful in this direction. An intriguing feature is that these materials are characterized by frustrated inter-units microscopic couplings. Nevertheless, the INS predictions suggest that their essential physics can be captured by means of a spin model where the original frustrated inter-units couplings are replaced by FM ones. A zero-temperature numerical study of this effective simplified spin model has been performed in Ref. [9] and the results are in agreement with previous conventional investigations [15].

Effective inter-units FM couplings may also arise from different mechanisms. An important example has been considered in Ref. [11] where a unified picture of recent INS susceptibility data [16] for stripe-ordered $L a_{15 / 8} \mathrm{Ba}_{1 / 8} \mathrm{CuO} \mathrm{O}_{4}$ is given based on a model of two-legs spin ladders where an effective FM interladder coupling results from integrating out the degrees of freedom in the stripes.

Support to the ferromagnetically coupled structural spin units scenario is given also by first-principles and Monte Carlo calculations of electronic structures about the nature of the spin-singlet ground state in the dimer compound $\mathrm{CaCuGe}_{2} \mathrm{O}_{6}$ [14]. The results obtained for the susceptibility and magnetization behavior give indeed evidence of effective FM inter-dimer couplings and of long-range interactions effects. In addition there are indications [9] of compounds which consist of ferromagnetically coupled ladders, as $\mathrm{SrCu}_{2} \mathrm{O}_{3}$ [17].

To gain insight into the properties of spin materials with weak FM interunits couplings, also in the presence of a magnetic field, the original complex spin Hamiltonian is conveniently mapped in an effective spin-1/2 FM XXZ model with spins, exchange couplings and longitudinal magnetic field expressed perturbatively as linear combinations of the original microscopic 
ones $[18,7,8,9,10]$. The final Hamiltonian has the general structure

$$
H=-\sum_{i, j=1}^{N}\left[J_{i j}\left(S_{i}^{x} S_{j}^{x}+S_{i}^{y} S_{j}^{y}\right)+K_{i j} S_{i}^{z} S_{j}^{z}\right]-h \sum_{i=1}^{N} S_{i}^{z}
$$

where $S_{i}^{\alpha}(\alpha=x, y, z)$ denote the effective spin components at site $i$ (which is a spin unit index in the original spin model) of a lattice with $\mathrm{N}$ sites and $J_{i j}>0, K_{i j}>0\left(\right.$ with $\left.J_{i i}=K_{i i}=0\right)$.

A systematic study of the thermodynamics of weakly ferromagnetically coupled spin units was performed [18] more than three decades ago within a mean field approximation (MFA). Further few studies for specific problems at zero temperature and, in some cases, in absence of a magnetic field, have been achieved at numerical, Hartree-Fock and MFA levels $[7,8,9,10,14,18]$. However, reliable experimental and theoretical studies of the magnetic properties and of the phase boundary of these systems close to the field-induced QCP are still lacking at the present time.

The aim of this letter is to give a contribution in this direction by using an appropriate functional representation of the FM spin model (1) $[19,20]$ and a wilsonian renormalization group $(R G)$ approach, which is the most valid and reliable tool to take properly into account fluctuations effects close to quantum and classical critical points. For generality purposes and with the intent to interpret some experimental findings [21,22], we refer to a $d$ dimensional spin lattice and include the possibility of long-range FM couplings which decrease with the distance between the spins as a power law of the type $r_{i j}^{-(d+\sigma)}$, with $\sigma \leq 2$. The value $\sigma=2$ corresponds to nearest-neighbor FM spin-spin interactions.

The action appropriate to describe the low-temperature properties of the FM 
model (1) was derived almost three decades ago [19] and sounds as

$$
\begin{aligned}
& S\left\{\psi^{*}, \psi\right\}=\sum_{q}\left(r_{0}+k^{\sigma}-i \omega_{l}\right)|\psi(q)|^{2}+ \\
& \frac{T_{0}}{4 V} \sum_{\left\{q_{\nu}\right\}} U\left(u_{0}, v_{0} ;\left\{q_{\nu}\right\}\right) \psi^{*}\left(q_{1}\right) \psi^{*}\left(q_{2}\right) \psi\left(q_{3}\right) \psi\left(q_{4}\right)
\end{aligned}
$$

where $q \equiv\left(\vec{k}, \omega_{l}\right)$ and $U\left(u_{0}, v_{0} ;\left\{q_{\nu}\right\}\right)=\delta_{\vec{k}_{1}+\vec{k}_{2} ; \vec{k}_{3}+\vec{k}_{4}}\left(u_{0} \delta_{\omega_{l_{1}}+\omega_{l_{2}} ; \omega_{l_{3}}+\omega_{l_{4}}}+v_{0} \delta_{\omega_{l_{1}} ; \omega_{l_{3}}} \delta_{\omega_{l_{2}} ; \omega_{l_{4}}}\right)$. Here, $\psi\left(\vec{k}, \omega_{l}\right)$ is a complex field related to the in-plane magnetization, $\vec{k}$ denote the wave vectors (we assume a cut-off $\Lambda=1$ related to the original spin lattice $), \omega_{l}=2 \pi l T_{0}(l=0, \pm 1, \pm 2, \ldots)$ are the bosonic Matsubara frequencies and $\mathrm{V}$ is the volume of the system. In Eq. (2) $T_{0} \propto T$ ( $T$ is the physical temperature), $r_{0} \propto h-[J(0)-K(0)], v_{0} \propto K(0)$, where $J(0)$ and $K(0)$ are the $(\vec{k}=0)$-Fourier transforms of $J_{i j}$ and $K_{i j}$ in Eq. (1). The explicit expressions of $T_{0}$ and $r_{0}, u_{0}, v_{0}$ in terms of the coupling parameters in the Hamiltonian (1), inessential for present purposes, can be found in Ref. [19]. Of course, if we put $v_{0}=0\left(K_{i j}=0\right.$ in Eq. (1) $), d=3$ and $\sigma=2$, the action (2) reduces to the well-known one for a 3-dimensional XY model in a transverse field [23] and for a dilute gas of hard-core bosons with chemical potential $\mu=-r_{0}$, which is believed $[24,25,26]$ to be appropriate for a description of the dimer compounds $\mathrm{XCuCl}_{3}(X=K, T l)$ close to their QCP's.

Applying the Wilson RG transformation to the action (2) and working to 
one-loop approximation we obtain the equations

$$
\begin{aligned}
& \frac{d r}{d l}=\sigma r+\frac{K_{d}}{2}\left[(v+2 u) F_{1}(r, T)+\frac{v T}{1+r}\right] \\
& \frac{d u}{d l}=(\sigma-d) u-\frac{K_{d}}{2} u^{2}\left[4 F_{2}(r, T)+F_{3}(r, T)\right]-\frac{5}{2} K_{d} \frac{u v T}{(1+r)^{2}} \\
& \frac{d v}{d l}=(\sigma-d) v-\frac{K_{d}}{2}\left(v^{2}+4 u v\right) F_{2}(r, T)-\frac{3}{2} K_{d} \frac{v^{2} T}{(1+r)^{2}} \\
& \frac{d T}{d l}=\sigma T\left(1-\frac{K_{d}}{2 \sigma} \frac{v T}{(1+r)^{2}}\right)
\end{aligned}
$$

for which the appropriate Fisher exponent is $\eta=2-\sigma$. In Eqs. (3), $l$ denotes the RG rescaling parameter, $F_{1}(r, T)=(1 / 2) \operatorname{coth}[(1+r) / T], F_{2}(r, T)=$ $-\partial F_{1}(r, T) / \partial r$ and $F_{3}(r, T)=F_{1}(r, T) /(1+r)$.

The $\left(T_{0}=0\right)$-critical properties can be simply obtained setting $T=0$ in Eqs. (3). For case of interest $d>\sigma$, when the quantum gaussian fixed point is stable, one finds MFA criticality in terms of $\left(r_{0}-r_{0 c}\right) \propto\left(h-h_{c}\right)$ as $r_{0} \rightarrow r_{0 c}^{+}$, where $r_{0 c}=-\left(K_{d} / 4 d\right)\left(v_{0}+2 u_{0}\right)$ localizes the QCP. Of course, the corresponding $\left(T_{0}=0\right)$-critical value $h_{c}$ of $h$ can be easily found from the explicit expressions of $r_{0}, u_{0}, v_{0}$ in terms of the original coupling parameters.

The wilsonian classical critical regime at finite temperature for $d<2 \sigma$ can be analyzed defining $\tilde{u}=u T, \tilde{v}=v T$, and then setting $T(l) \rightarrow \infty$ as $l \rightarrow \infty$ in the RG equations for the new coupling parameters $\tilde{u}$ and $\tilde{v}$.

Here, we are interested to solve Eqs. (3) for $\sigma<d<2 \sigma$ limiting ourselves to quantum or classical gaussian regime. This can be performed in the lowtemperature limit and working to leading order in the coupling parameters.

With $u(l) \simeq u_{0} e^{-(d-\sigma) l}, v(l) \simeq v_{0} e^{-(d-\sigma) l}$ and $T(l) \simeq e^{\sigma l} T_{0}(z=\sigma$ is the appropriate dynamical critical exponent), $r(l)$ is obtained through the non- 
linear relevant scaling field $g(l)=r(l)+\left(K_{d} / 4 d\right)(v(l)+2 u(l))$ which scales as

$$
\begin{aligned}
g(l) & =e^{\sigma l}\left\{g_{0}+\frac{K_{d} v_{0} T_{0}}{2(d-\sigma)}\left(1-e^{-(d-\sigma) l}\right)+\right. \\
& \left.+\frac{K_{d}}{2 \sigma}\left(v_{0}+2 u_{0}\right) T_{0}^{\frac{d}{\sigma}} \int_{0}^{T(l)} d y \frac{y^{-\frac{d+\sigma}{\sigma}}}{e^{\frac{1}{y}}-1}\right\},
\end{aligned}
$$

with $g_{0}=r_{0}-r_{0 c}$.

We now stop the renormalization procedure at a scale $l^{*} \gg 1$ to be determined setting $g\left(l^{*}\right) \simeq 1$. Then, from Eq. (4), with $T_{0} \ll 1$ but arbitrary $T\left(l^{*}\right)=$ $e^{\sigma l^{*}} T_{0}$, we have for the dimensionless inverse susceptibility $\mathbf{x}=\left(\chi / \chi_{0}\right)^{-1}=$ $e^{-\sigma l^{*}}$, the self-consistent equation

$$
\begin{aligned}
\mathrm{x} & =g_{0}+\frac{K_{d} v_{0} T_{0}}{2(d-\sigma)}\left(1-\mathrm{x}^{\frac{d-\sigma}{\sigma}}\right)+ \\
& +\frac{K_{d}}{2 \sigma}\left(v_{0}+2 u_{0}\right) T_{0}^{d / \sigma} \int_{0}^{T_{0} / \mathrm{x}} d y \frac{y^{-\frac{d+\sigma}{\sigma}}}{e^{\frac{1}{y}}-1}
\end{aligned}
$$

which contains all the relevant physical information and allows us to explore the full quantum critical region in the phase diagram for $d>\sigma$.

The phase boundary equation $r_{0 c}\left(T_{0}\right)$ in the $\left(r_{0}, T_{0}\right)$-plane, ending in the QCP, can be obtained setting $\mathrm{x}=0\left(l^{*}=\infty\right)$ in Eq. (5). One obtains

$$
\begin{aligned}
g_{0 c}\left(T_{0}\right) & =r_{o c}\left(T_{0}\right)-r_{o c}= \\
& -\frac{K_{d} v_{0} T_{0}}{2(d-\sigma)}-\frac{K_{d} A_{d, \sigma}}{2 \sigma}\left(v_{0}+2 u_{0}\right) T_{0}^{d / \sigma}
\end{aligned}
$$

with $A_{d, \sigma}=\Gamma(d / \sigma) \zeta(d / \sigma)$. It is worth noting that, if one assumes $v_{0}=0$, we have $r_{0 c}\left(T_{0}\right)-r_{0 c} \propto \Delta H_{c}(T)=h_{c}(T)-h_{c} \propto T^{d / \sigma}$ and hence the result $\psi=d / \sigma$ for the phase boundary or shift exponent defined as $\Delta H_{c}(T) \propto T^{\psi}$. For $d=3$ and $\sigma=2$, we find, as expected [24,25], $\psi=3 / 2$. In contrast, with $v_{0} \neq 0$ 
and for sufficiently low temperature, Eq. (6) yields $\psi=1$ which is a universal value independent of $d$ and the interaction parameter $\sigma$. Thus, the structure of the phase boundary equation (6) close to the QCP suggests the existence of a crossover temperature $T_{0}^{*}$ between two different regimes characterized by the values $d / \sigma$ and 1 of the exponent $\psi$ decreasing the temperature. By inspection of Eq. (6), one immediately sees that (with small $S^{z}-S^{z}$ coupling)

$$
T_{0}^{*}=\left[\frac{\sigma v_{0}}{(d-\sigma) A_{d, \sigma}\left(v_{0}+2 u_{0}\right)}\right]^{\frac{\sigma}{d-\sigma}} \sim[K(0)]^{\frac{\sigma}{d-\sigma}}
$$

and the mentioned crossover is described by the effective exponent

$$
\psi_{e f f}(\tau)=\frac{\partial \ln g_{0 c}(\tau)}{\partial \ln \tau}=\frac{1+\left(\frac{d}{\sigma}\right) \tau^{\frac{d-\sigma}{\sigma}}}{1+\tau^{\frac{d-\sigma}{\sigma}}},
$$

with $\tau=T_{0} / T_{0}^{*}$ and $1 \leq \psi_{\text {eff }}(\tau) \leq d / \sigma$. For case $d=3$ and $\sigma=2$, Eq. (8) yields $\psi_{\text {eff }}(\tau)=\left[1+(3 / 2) \tau^{1 / 2}\right] /\left(1+\tau^{1 / 2}\right)$, with $1 \leq \psi_{\text {eff }} \leq 3 / 2$.

Although all the quantum critical properties in the gaussian region close to the QCP can be obtained solving the self-consistent Eq. (5), we limit ourselves to the experimentally relevant case $r_{0}=r_{0 c}\left(h=h_{c}\right)$ as $T_{0} \rightarrow 0$. A solution exists for $T_{0} e^{\sigma l^{*}}=T\left(l^{*}\right) \gg 1$ and we find

$$
\begin{aligned}
& e^{-\sigma l^{*}} \simeq \frac{K_{d} v_{0}}{2(d-\sigma)} T_{0}+\frac{K_{d} A_{d, \sigma}}{2 \sigma}\left(v_{0}+2 u_{0}\right) T_{0}^{\frac{d}{\sigma}} \\
& \approx\left\{\begin{array}{l}
\frac{K_{d} v_{0}}{2(d-\sigma)} T_{0}, \quad T_{0}<T_{0}^{*} \\
\frac{K_{d} A_{d, \sigma}}{2 \sigma}\left(v_{0}+2 u_{0}\right) T_{0}^{\frac{d}{\sigma}}, \quad T_{0}>T_{0}^{*} .
\end{array}\right.
\end{aligned}
$$

This implies that also for susceptibility $\chi \sim e^{\sigma l^{*}}$ and correlation length $\xi \sim$ $\chi^{1 / \sigma} \sim e^{l^{*}}$ one can define effective exponents yielding $\gamma_{\text {eff }}(\tau)=\psi_{\text {eff }}(\tau)$ and $\nu_{\text {eff }}(\tau)=(1 / \sigma) \psi_{\text {eff }}(\tau)$. For the realistic values $d=3$ and $\sigma=2$, one has $\left(\chi \sim T_{0}^{-1}, \xi \sim T_{0}^{-1 / 2}\right)$ and $\left(\chi \sim T_{0}^{-3 / 2}, \xi \sim T_{0}^{-3 / 4}\right)$ for $T_{0}<T_{0}^{*}$ and $T_{0}>T_{0}^{*}$, 
respectively. The low- $T_{0}$ behaviors of other quantities can be obtained using the standard RG machinery.

The previous results, and in particular the phase boundary exponent $\psi$ (or, more properly, $\psi_{\text {eff }}(\tau)$ in our RG scenario), may constitute a good basis for comparisons with available and possible future experimental data. Indeed our RG analysis suggests that, close to the QCP, one must expect boson-like behavior $(\psi=d / \sigma)$ only above a characteristic temperature $T^{*} \propto T_{0}^{*}\left(v_{0}, u_{0}\right)$ depending on the coupling parameters in the original effective FM XXZ spin model. Decreasing the physical temperature below $T^{*}$, a crossover takes place to a different regime where the phase boundary is characterized by a different universal exponent $\psi=1$. Of course, the amplitude of the crossover region reduces to zero decreasing $v_{0}$. In particular, when $v_{0}=0$ or negligibly small, only the exponent $\psi=d / \sigma$ should be observable. In contrast, if the crossover region is sufficiently large, only the linear behaviour in $T$ is expected at sufficiently low temperature.

A situation of this type seems to occur for the doped dimer compound $T l_{1-x} K_{x} C u C l_{3}$ for which the effect of randomness on field induced magnetic ordering has been recently investigated through low-temperature specific heat measurements for potassium concentration in the interval $0 \leq x<0.22$ [27]. The experimental predictions for the phase boundary exponent $\psi$, relevant for our purposes, can be summarized as follows.

First, a reevaluation of $\psi$ for $x=0$ yields the value $\psi=1.67$ which is close to $\psi_{\mathrm{BEC}}=3 / 2$ derived, for compound $\mathrm{TlCuCl}_{3}$, from the theory of Bose-Einstein condensation of triplet excitations.

Next, a systematic study for $x \neq 0$ shows that the disorder produces a sensible 
change in the critical scenario. In particular, the phase boundary is accurately measured for temperature below $2 \mathrm{~K}$ and the relevant features are:

i) the exponent $\psi$ decreases systematically increasing $x$ from the $(x=0)$-value $\psi=1.67$ and tends to $\psi=1$ for $x>0.1$. This corresponds to an enlargement of the phase boundaries obtained for different values of $x$;

ii) the phase boundary observed for $x>0.1$ is almost a linear function of temperature. These findings are clearly shown in Fig. 4 of Ref. [27].

To explain these results, it was conjectured in Ref. [27] that a Bose glass of triplons, in the sense argued by Fisher et al. [28], could be an appropriate model to describe properly the quantum criticality of $\mathrm{Tl}_{1-x} K_{x} C u C l_{3}$ with $x \neq 0$. Nevertheless, the Bose glass theory, as applied to this compound close to the QCP, is expected to predict [27] a phase boundary exponent $\psi \leq 1 / 2$ as $T \rightarrow 0$, in drastic disagreement with the available measurements. To solve this puzzle it was also suggested that a crossover from the convex form $(\psi>1)$ to the concave form $(\psi<1)$ of the phase boundary should occur very close to the QCP. However, this type of crossover is not observed in the experiment and hence the problem remains an open question.

The results here obtained allow us to speculate that the enlargement of the phase boundaries and the almost linear dependence of the critical field on temperature for $x>0.1$ [27] can be simply interpreted in terms of the previous $\mathrm{RG}$ scenario involving the characteristic crossover temperature $T^{*} \sim v_{0}^{\sigma /(d-\sigma)}$ with $\sigma=2$ and $d=3$. To show this, we adopt for $T l_{1-x} K_{x} C u C l_{3}$ the idea that the doping, as frustration, induces an effective FM coupling between spin dimers. Furthermore we assume, reasonably, that $v_{0}$, and hence $T^{*}$, vanishes or its effect becomes negligible when $x$ decreases to zero (this lies on the feature that the action (2), with $v_{0}=0$, appears to work for the dimer compound 
$\left.\mathrm{TlCuCl}_{3}[24,25]\right)$.

For $x=0$ our analysis predicts that the phase boundary behavior for $T \rightarrow 0$ is characterized by the Bose exponent $\psi=3 / 2$, as expected for $\mathrm{TlCuCl}_{3}[26]$. For $x \neq 0$, the effective FM $S^{z}-S^{z}$ coupling in the Hamiltonian (1) becomes active and a crossover is expected to occur from $\psi=3 / 2$ to $\psi=1$ decreasing the temperature to zero across $T^{*}$. When $x \lesssim 0.1$ [27], the "temperature window" $\left(0, T^{*}\right)$ starts to be experimentally accessible and a $\psi \lesssim 3 / 2$ is measured. Increasing $x$ (above 0.1), this window becomes larger and larger, the temperature can be decreased to zero working below $T^{*}$ and one recovers the exponent $\psi=1$ which characterizes the linear temperature behavior of the phase boundary observed in the experiment close to the QCP. Besides, since for $T \rightarrow 0$ it is found $3 / 2 \geq \psi \geq 1$ increasing $x$, one can argue that an enlargement of the phase boundaries takes place, again in agreement with the experimental data [27].

From our RG predictions one can obtain also some insight into the $x$-dependence of the crossover temperature $T^{*}$ as given by Eq. (7) in terms of the effective longitudinal spin coupling $K(0)$, with $v_{0} \propto K(0)$ [19]. Indeed, since one expects that $v_{0}=0$ for $x=0[24,25]$, we can assume plausibly that $v_{0}(x) \propto K(0) \sim x$ for small potassium concentration. Hence, Eq. (7) yields $T^{*} \sim x^{\sigma /(d-\sigma)}$ and, for $d=3$ and $\sigma=2$, one has $T^{*}(x) \sim x^{2}$, result which could be tested experimentally. From a theoretical point of view, these results should be extracted from an appropriately chosen effective spin dimer model and then using the explicit relations between the bare couplings and the effective FM ones obtained by mapping [18] the original Hamiltonian in the FM XXZ model (1). A study of this not easy problem is in progress and we plan to present a quantitative scenario in a future work. 
In any case, further reliable experimental and theoretical studies on ferromagnetically coupled structural spin units are desirable and we hope that this preliminary contribution will stimulate more intensive research activity on the subject.

\section{References}

[1] S. Sachdev, Quantum Phase Transitions, Cambridge University Press, Cambridge (1999).

[2] W. Shiramura et al., J. Phys. Soc. Jpn. 66, 1900 (1997); A. Oosawa et al., Phys. Rev. B 66, 104405 (2002).

[3] A. Oosawa, M. Ishii, and H. Takada, J. Phys. Condens. Matter 11, 265 (1999); Ch. Rüegg et al., Nature 423, 62 (2003).

[4] M. Jaime et al., Phys. Rev. Lett. 93, 087203 (2004).

[5] G. Chaboussant et al., Phys. Rev. B 55, 3046 (1997); M. Mito et al., Phys. Rev. 65, 104405 (2002), and references therein.

[6] E. Dagotto, Rep. Progr. Phys. 62, 1525 (1999); K. Hijii, A. Kitazawa, and K. Nomura, Phys. Rev. B 72, 014449 (2005).

[7] B. Grenier et al., Phys. Rev. Lett. 92, 177202 (2004).

[8] F. Mila, Eur. Phys. J. B 6, 201 (1998).

[9] S. Dalosto and J. Riera, Phys. Rev. B 62, 928 (2000), and references therein.

[10] F. Zhou, M. Snock, J. Wiemer, and I. Affleck, Phys. Rev. B 70, 184434 (2004).

[11] G. S. Uhrig, K. P. Schmidt and M. Grüninger, Phys. Rev. Lett. 93, 267003 (2004). 
[12] L. P. Regnault et al., Phys. Rev. B 59, 1055 (1999).

[13] T. Kato et al., J. Phys. Soc. Jpn. 67, 752 (1998).

[14] R. Valenti, T. Saha-Dasgupta, and C. Gros, Phys. Rev. B 66, 054426 (2002).

[15] B. Normand, k. Penc, M. Albrech, and F. Mila, Phys. Rev. B56, 5736 (1997);

S. Miyahara, M. Troyer, D. C. Johnston, and K. Ueda, J. Phys. Soc. Jpn. 67, 3918 (1998).

[16] For recent reviews see: Y. Sidis et al., Phys. Status Solidi B 241, 1204 (2004); M. R. Norman and C. Pépin, Rep. Progr. Phys. 66, 1547 (2003).

[17] M. Azuma et al., Phys. Rev. Lett. 73, 3463 (1994); C. de Graaf, I. de P. R. Moreira, F. Illas and R. L. Martin, Phys. Rev. B60, 3457 (1999).

[18] M. Tachiki and T. Yamada, J. phys. Soc. Jpn. 28, 1413 (1970).

[19] D. K. Dacol, J. Low Temp. Phys. 41, 349 (1980).

[20] A. Caramico D'Auria, L. De Cesare, M. T. Mercaldo, and I. Rabuffo, Physica A 351, 294 (2005).

[21] H. Tsujii et al., cond-mat / 0509169.

[22] O. Nohadani, S. Wessel, B. Normand, and S. Haas, Phys. Rev. B 69, 220402(R) (2004).

[23] P. B. Gerber, H. Beck, J. Phys. C : Solid State Phys. 10, 4013 (1977).

[24] See the recent review N. Kawashima, J. Phys. Soc. Jpn. 74, 145 (2005).

[25] N. Kawashima, J. Phys. Soc. Jpn. 73, 3219 (2004).

[26] Wwhen $v_{0} \neq 0$, this action is no more adequate to describe the quantum criticality of an AFM XXZ model [19].

[27] Y. Shindo and H. Tanaka, J.Phys. Soc. Jpn. 73, 2642 (2004). 
[28] M.P.A. Fisher, P.B. Weichman, G. Grinstein, and D.S. Fisher, Phys. Rev. B 40, 546 (1989). 